\title{
THE EXISTENCE OF HORIZONS IN AN ASYMPTOTICALLY FLAT 3-MANIFOLD
}

\author{
YU YAN
}

\begin{abstract}
In this paper we study the type of asymptotically flat manifolds of the form $\left(S^{3} \backslash\{P\}, G^{4} g\right)$, where $G$ is a Green's function of the conformal Laplacian of $\left(S^{3}, g\right)$ at $P$. We give a sufficient condition on the background metric $g$ which guarantees the existence of a minimal surface in $\left(S^{3} \backslash\{P\}, G^{4} g\right)$.
\end{abstract}

\section{Introduction}

In general relativity a spacetime is a 4 dimensional manifold with a Lorentz metric $\bar{g}$ which satisfies the Einstein's equation

$$
G:=\operatorname{Ric}(\bar{g})-\frac{1}{2} R(\bar{g}) \cdot \bar{g}=T
$$

where $\operatorname{Ric}(\bar{g})$ and $R(\bar{g})$ are the Ricci curvature tensor and the scalar curvature of $\bar{g}$, respectively, $T$ is the energy-momentum tensor, and $G$ is called the Einstein curvature tensor. Let $(M, \bar{g})$ be a spacetime, an initial data set is a 3 dimensional manifold $N$ which is a spacelike time slice of $M$, with a positive definite metric $g_{i j}$, a symmetric tensor $p_{i j}$ (second fundamental form of $N$ in $M$ ), a local mass density $\mu$, and a local current density $J^{i}$. The constraint equations which determine whether $N$ is a spacelike hypersurface in $(M, \bar{g})$ with second fundamental form $p_{i j}$ are given by

$$
\begin{aligned}
\mu & =\frac{1}{2}\left(R-\operatorname{Tr} p+\left(\sum_{i} p_{i}^{i}\right)^{2}\right) \\
J^{i} & =\sum_{j} D_{j}\left(p^{i j}-\left(\sum_{k} p_{k}^{k}\right) g^{i j}\right) .
\end{aligned}
$$

We assume that $\mu$ and $J^{i}$ obey the dominant energy condition

$$
\mu \geq\left(\sum_{i} J^{i} J_{i}\right)^{\frac{1}{2}}
$$

Received December 23, 2004. 
A very interesting question in the study of general relativity is the existence of black holes, which are the regions where gravitation is so strong that no particle or light entering that region can escape from it. We would like to determine the existence and location of a black hole, and since sometimes we only have the information about an initial data set of a spacetime, it would be useful if such criteria don't require the knowledge of the global time development of the spacetime. One well-known such a criterion was given by Hawking and Penrose which states that under some physical assumptions, the apparent horizon must lie in a black hole, where an apparent horizon is a 2 -sphere $\Sigma$ in the initial data set whose mean curvature $H$ satisfies $H=\operatorname{Tr}_{\Sigma} p$.

There are some interesting results on the existence of apparent horizons. For example, R. Schoen and S.T. Yau [7] obtained an existence theorem under the assumption of large mass density for mass fields by studying Jang's equation

$$
\sum_{i, j}\left(p^{i j}-\frac{D^{i} D^{j} f}{\left(1+|\nabla f|^{2}\right)^{\frac{1}{2}}}\right)\left(g_{i j}-\frac{D_{i} f D_{j} f}{1+|\nabla f|^{2}}\right)=0 .
$$

Later also from Jang's equation Eardley [2] proved another existence result when the area-to-volume ratio in some region is sufficiently small.

We look at the simplified case in which the spacetime is vacuum $(T=0)$ and the initial data set $\left(N, g_{i j}, p_{i j}\right)$ is totally geodesic in the spacetime, i.e., it satisfies $p=0$. Under these assumptions, since $\mu=T_{00}=0$, we know from (2) that the scalar curvature $R(g)$ of $N$ is 0 . The apparent horizon equation now becomes $H=0$, i.e, an apparent horizon is just a minimal surface in $N$.

We also assume that the initial data set is asymptotically flat, which is defined as the following:

Definition 1.1. $\left(M^{n}, g\right)$ is said to be asymptotically flat if there is a compact set $K \subset M$ and a diffeomorphism $\Phi: M-K \rightarrow \mathbf{R}^{n}-\{|x|<1\}$ such that, in the coordinate chart defined by $\Phi$,

$$
g=\sum_{i j} g_{i j}(x) d x^{i} d x^{j}
$$

where

$$
\begin{aligned}
g_{i j}(x) & =\delta_{i j}+O\left(|x|^{-p}\right) \\
|x|\left|g_{i j, k}(x)\right|+|x|^{2}\left|g_{i j, k l}(x)\right| & =O\left(|x|^{-p}\right) \\
|R(g)| & =O\left(|x|^{-q}\right)
\end{aligned}
$$

for some $p>\frac{n-2}{2}$ and some $q>n$, where we have used commas to denote partial derivatives in the coordinate chart, and $R(g)$ is the scalar curvature of $\left(M^{n}, g\right)$.

We can construct a scalar flat and asymptotically flat manifold by conformally deforming the metric on a compact manifold using the Green's function:

Let $(M, g)$ be a 3 -dimensional compact manifold with positive scalar curvature $R(g)>0$. Choose a point $P \in M$, since $R(g)>0$ the Green's function $G$ of the 
conformal Laplacian $8 \Delta_{g}-R(g)$ at $P$ exists. Then $G^{4} g$ is a metric defined on $M \backslash\{P\}$ and conformal to $g$.

On $M \backslash\{P\}, R\left(G^{4} g\right)=-G^{-\frac{n+2}{n-2}}\left(8 \Delta_{g} G-R(g) G\right)=0$, so the manifold $(M \backslash$ $\left.\{P\}, G^{4} g\right)$ is scalar flat.

By direct calculation it can also be shown that $\left(M \backslash\{P\}, G^{4} g\right)$ is an asymptotically flat manifold.

The case we are interested in is $M=S^{3}$. For manifolds not homeomorphic to $S^{3}$, there must be a stable minimal surface on $M$ for topological reasons, so the problem of finding minimal surfaces on $\left(M \backslash\{P\}, G^{4} g\right)$ is less interesting.

We make the following assumptions on $\left(S^{3}, g\right)$ : the diameter of $\left(S^{3}, g\right) \leq D$, the total volume of $\left(S^{3}, g\right)=V_{T} \geq V$ and the Ricci curvature has a (possibly negative) lower bound $\operatorname{Ric}(g) \geq \mu$.

The main result in this paper is:

Theorem 1.2. For any $r>\frac{3}{2}$, there exists $0<\delta=\delta(V, D, \mu, r) \leq 1$ such that if $\|R(g)\|_{L^{r}\left(S^{3}\right)}<\delta$ and $R(g)>0$, then the asymptotically flat manifold $\left(S^{3} \backslash\right.$ $\left.\{P\}, G^{4} g\right)$ contains a horizon, where $G$ is the Green's function of the conformal Laplacian $8 \triangle_{g}-R(g)$ at $P$.

There do exist metrics which satisfy the conditions in this existence theorem. By a theorem of Kazdan and Warner [3] there exists a negative scalar curvature metric on any compact Riemannian manifold. Then we can construct a continuous path of metrics from a negative scalar curvature metric on $S^{3}$ which has negative Yamabe invariant to one with positive Yamabe invariant. Since the Yamabe invariant changes continuously, we can find a metric on the path such that its Yamabe invariant is 0 . Then there exists a metric $g$ conformal to that metric with $R(g)=0$, we can assume $g$ has total volume 1, i.e. $\int_{M} d v_{g}=1$. We claim that there exists a sequence of metrics $\left\{g_{i}\right\}$ such that $R\left(g_{i}\right)>0$ and $g_{i} \rightarrow g$. Then $g_{i}$ has uniform lower and upper volume bounds, upper diameter bound and lower Ricci bound, and since $R\left(g_{i}\right) \rightarrow R(g)=0, g_{i}$ will satisfy the condition in the theorem for sufficiently large $i$. To prove the claim, consider the family of metrics $\bar{g}_{t}=g+t \operatorname{Ric}(g)$. It can be conformally deformed to a continuous family of metrics $g_{t}=u_{t}^{4} \bar{g}_{t}$ of constant scalar curvature and total volume 1. By this definition $g_{0}=\bar{g}_{0}=g$. Suppose $R\left(g_{t}\right) \leq 0$ for all $t$ near 0 , 
then $\left.\frac{d}{d t}\right|_{t=0} \int_{M} R\left(g_{t}\right) d v_{g_{t}}=0$. By an argument similar to that in [8],

$$
\begin{aligned}
0 & =\left.\frac{d}{d t}\right|_{t=0} \int_{M} R\left(g_{t}\right) d v_{g_{t}} \\
& =-\left.\int_{M}\left\langle\frac{\partial g_{t}}{\partial t}, \operatorname{Ric}\left(g_{t}\right)-\frac{1}{2} R\left(g_{t}\right) g_{t}\right\rangle_{g_{t}} d v_{g_{t}}\right|_{t=0} \\
& =-\left.\int_{M}\left\langle 4 u_{t}^{3} \frac{\partial u_{t}}{\partial t} \bar{g}_{t}+u_{t}^{4} \frac{\partial \bar{g}_{t}}{\partial t}, \operatorname{Ric}\left(g_{t}\right)-\frac{1}{2} R\left(g_{t}\right) g_{t}\right\rangle_{g_{t}} d v_{g_{t}}\right|_{t=0} \\
& =-\int_{M}\left\langle\left. 4 \frac{\partial u_{t}}{\partial t}\right|_{t=0} g+\operatorname{Ric}(g), \operatorname{Ric}(g)\right\rangle_{g} d v_{g} \\
& =-\int_{M}\langle\operatorname{Ric}(g), \operatorname{Ric}(g)\rangle_{g} d v_{g}
\end{aligned}
$$

since $\langle g, \operatorname{Ric}(g)\rangle=R(g)=0$. This then implies that $g$ is Ricci flat and hence flat since the dimension of the manifold is 3 . This is a contradiction, so the claim is true.

\section{Two Technical Lemmas}

The proof of Theorem 1.2 needs to use two technical lemmas, which are rather general. They can be applied to any manifold $\left(M^{n}, g\right)$ as long as for $B_{R} \subset M^{n}$ with $R$ appropriately small, we have

- Sobolev Inequality: there exists a constant $C_{1}$, such that

$$
\left(\int_{B_{R}}|f|^{\frac{n}{n-1}} d v_{g}\right)^{\frac{n-1}{n}} \leq C_{1} \int_{B_{R}}|\nabla f| d v_{g}
$$

for any $f \in C^{1}\left(B_{R}\right)$ with $f=0$ on $\partial B_{R}$.

- Weak Poincaré Inequality: there exist $C_{2}$ and $\theta_{1} \in(0,1]$ such that for any $R>0$ with $\overline{B_{R}}$ compact in $M$,

$$
\inf _{\beta \in \mathbf{R}} \int_{B_{\theta_{1} R}}|f-\beta|^{2} d v_{g} \leq C_{2} R^{2} \int_{B_{R}}|\nabla f|^{2} d v_{g}
$$

for any $f \in C^{1}\left(B_{R}\right)$.

- Upper Volume Bound: there exists a constant $C_{3}$, such that for any $R>0$,

$$
\operatorname{Vol}\left(B_{R}\right) \leq C_{3} R^{3} .
$$

Lemma 2.1. If $u>0$ is a supersolution of the equation $\triangle u-f u=h$ on $a$ geodesic ball $B_{R}$ and $f, h \in L^{r}\left(B_{R}\right)$ for some $r>\frac{n}{2}$, then there exists $p_{0}>0$ such that for any $0<p<p_{0}$ and $0<\theta \leq \theta_{1}$, u satisfies

$$
\inf _{B_{\theta R}} u+R^{2-\frac{n}{r}}\|h\|_{L^{r}\left(B_{R}\right)}>C\left(R^{-n} \int_{B_{R}} u^{p} d v_{g}\right)^{1 / p}
$$


where $C$ depends on $C_{1}, C_{2}, C_{3}, n, \theta, p, r, R$ and the upper bound on $\int_{B_{R}}|f|^{r} d v_{g}$; $p_{0}$ depends on $C_{1}, C_{2}, C_{3}, n, \theta, r, R$ and the upper bound on $\int_{B_{R}}|f|^{r} d v_{g}$.

Proof. Let $\delta=R^{2-\frac{n}{r}}\|h\|_{L^{r}\left(B_{R}\right)}$. Choose a $C^{2}\left(\mathbf{R}^{+}\right)$function $\phi$ satisfying the following conditions:

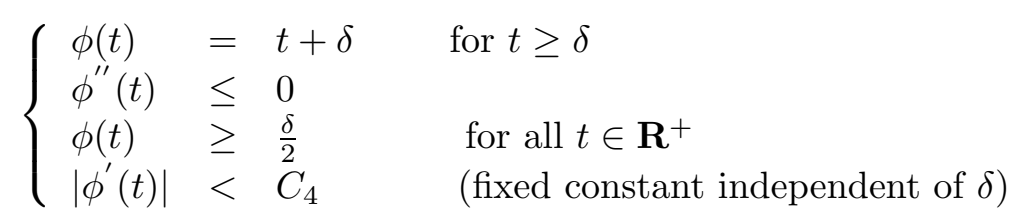

By this choice $t<\phi(t) \leq t+\delta$ for all $t \geq 0$.

For any $\xi \in C_{c}^{1}\left(B_{R}\right), \xi \geq 0$, by the definition of supersolutions we have

$$
\int_{B_{R}}\left[\nabla u \cdot \nabla\left(\xi \phi^{\prime}(u)\right)+f u\left(\xi \phi^{\prime}(u)\right)+h\left(\xi \phi^{\prime}(u)\right)\right] d v_{g} \geq 0 .
$$

Since $\phi^{\prime \prime}(u) \leq 0$, this implies

$$
\int_{B_{R}}\left[\nabla(\phi(u)) \cdot \nabla \xi+\left(\frac{f u \phi^{\prime}(u)}{\phi(u)}+\frac{h \phi^{\prime}(u)}{\phi(u)}\right) \phi(u) \xi\right] d v_{g} \geq 0 .
$$

By the choice of $\phi$,

$$
\left|\frac{f u \phi^{\prime}(u)}{\phi(u)}\right|<\left|C_{4} f\right| \quad \text { and } \quad\left|\frac{h \phi^{\prime}(u)}{\phi(u)}\right| \leq\left|\frac{2 C_{4} h}{\delta}\right| .
$$

Define $F=C_{4}|f|+\frac{2 C_{4}|h|}{\delta}$, then $\phi(u)$ is a supersolution of $\triangle-F=0$. The standard elliptic theory implies that there exists $p_{0}>0$ which depends on $C_{1}, C_{2}, C_{3}$ and the upper bound on $R^{2 r-n} \int_{B_{R}}|F|^{r} d v_{g}$, such that for all $0<p \leq p_{0}$

$$
\inf _{B_{\theta R}} \phi(u) \geq C\left(R^{-n} \int_{B_{R}} \phi(u)^{p} d v_{g}\right)^{\frac{1}{p}} \quad \text { for any } \quad \theta \leq \theta_{1}
$$

where $C$ is positive and depends on $n, \theta, p, r, R, C_{1}, C_{2}, C_{3}$ and the upper bound on $R^{2 r-n} \int_{B_{R}}|F|^{r} d v_{g}$. Note that

$$
\begin{aligned}
R^{2 r-n} \int_{B_{R}}|F|^{r} d v_{g} & =R^{2 r-n} \int_{B_{R}}\left(C_{4}|f|+\frac{2 C_{4}|h|}{\delta}\right)^{r} d v_{g} \\
& \leq 2^{r-1} C_{4}^{r}\left(R^{2 r-n} \int_{B_{R}}|f|^{r} d v_{g}+2^{r}\right)
\end{aligned}
$$

(by the definition of $\delta$ )

So $C$ depends on $n, \theta, p, r, R, C_{1}, C_{2}, C_{3}$ and the upper bound on $\int_{B_{R}}|f|^{r} d v_{g}$; $p_{0}$ depends on $n, \theta, r, R, C_{1}, C_{2}, C_{3}$ and the upper bound on $\int_{B_{R}}|f|^{r} d v_{g}$.

Since $u<\phi(u)<u+\delta$, by $(4)$

$$
\inf _{B_{\theta R}} u+\delta>C\left(R^{-n} \int_{B_{R}} u^{p} d v_{g}\right)^{\frac{1}{p}}
$$


Lemma 2.2. If $u>0$ is a subsolution of the equation $\triangle u-f u=h$ on a geodesic ball $B_{R}$ and $f, h \in L^{r}\left(B_{R}\right)$ for some $r>\frac{n}{2}$, then for any $p>0$ and $0<\theta<1$, $u$ satisfies

$$
\sup _{B_{\theta R}} u<C\left(R^{2-\frac{n}{r}}\|h\|_{L^{r}\left(B_{R}\right)}+\left(R^{-n} \int_{B_{R}} u^{p} d v_{g}\right)^{1 / p}\right)
$$

where the constant $C$ depends on $C_{1}, C_{3}, n, \theta, p, r, R$ and the upper bound on $\int_{B_{R}}|f|^{r} d v_{g}$.

Proof. The proof is very similar to that of Lemma 2.1. Let $\delta=R^{2-\frac{n}{r}}\|h\|_{L^{r}\left(B_{R}\right)}$. This time we choose $\phi(t) \in C^{2}\left(\mathbf{R}^{+}\right)$which satisfies

$$
\left\{\begin{array}{l}
\phi(t)=t \quad \text { for } t \geq \delta \\
\phi^{\prime \prime}(t) \geq 0 \\
\phi(t) \geq \frac{\delta}{2}=\phi(0)
\end{array}\right.
$$

Note that by this choice $\phi^{\prime}(t) \leq 1, \phi(t)>0$ and $t \leq \phi(t) \leq t+\frac{\delta}{2}$ for all $t \geq 0$.

For any $0 \leq \xi \in C_{c}^{1}\left(B_{R}\right)$, by the definition of subsolutions we have

$$
\int_{B_{R}}\left[\nabla u \cdot \nabla\left(\xi \phi^{\prime}(u)\right)+f u\left(\xi \phi^{\prime}(u)\right)+h\left(\xi \phi^{\prime}(u)\right)\right] d v_{g} \leq 0
$$

So

$$
\int_{B_{R}}\left[\nabla(\phi(u)) \cdot \nabla \xi+\left(\frac{f u \phi^{\prime}(u)}{\phi(u)}+\frac{h \phi^{\prime}(u)}{\phi(u)}\right) \phi(u) \xi\right] d v_{g} \leq 0,
$$

since $\phi^{\prime \prime}(u) \geq 0$.

By the choice of $\phi$,

$$
\left|\frac{f u \phi^{\prime}(u)}{\phi(u)}\right| \leq|f| \quad \text { and } \quad\left|\frac{h \phi^{\prime}(u)}{\phi(u)}\right| \leq\left|\frac{2 h}{\delta}\right| .
$$

Define $F=|f|+\frac{2|h|}{\delta}$, then

$$
\int_{B_{R}}[\nabla(\phi(u)) \cdot \nabla \xi-F \phi(u) \xi] d v_{g} \leq 0
$$

i.e. $\phi(u)$ is a subsolution of $\triangle+F=0$. The standard elliptic theory implies that for any $p>0$, there exists a constant $C^{\prime}$ which depends on $C_{1}, n, \theta, p$ and 
the upper bound on $R^{2 r-n} \int_{B_{R}}|F|^{r} d v_{g}$, such that

$$
\begin{aligned}
\sup _{B_{\theta R}} u & \leq \sup _{B_{\theta R}} \phi(u) \\
& \leq C^{\prime}\left(R^{-n} \int_{B_{R}} \phi(u)^{p} d v_{g}\right)^{\frac{1}{p}} \\
& \leq C^{\prime}\left(R^{-n} \int_{B_{R}}\left(\frac{\delta}{2}+u\right)^{p} d v_{g}\right)^{\frac{1}{p}} \\
& \leq C\left(\delta+\left(R^{-n} \int_{B_{R}} u^{p} d v_{g}\right)^{\frac{1}{p}}\right) \\
& =C\left(R^{2-\frac{n}{r}}\|h\|_{L^{r}\left(B_{R}\right)}+\left(R^{-n} \int_{B_{R}} u^{p} d v_{g}\right)^{\frac{1}{p}}\right)
\end{aligned}
$$

where $C$ depends on $C_{3}, r$ and $C^{\prime}$. Same argument as that in the proof of Lemma 2.1 shows that

$$
R^{2 r-n} \int_{B_{R}}|F|^{r} d v_{g} \leq 2^{r-1}\left(R^{2 r-n} \int_{B_{R}}|f|^{r} d v_{g}+2^{r}\right) .
$$

So $C$ depends on $C_{1}, C_{3}, n, \theta, p, r, R$ and the upper bound on $\int_{B_{R}}|f|^{r} d v_{g}$.

\section{Proof of the Existence Theorem}

We first give the idea of the proof. By a Harnack inequality type estimate, when $\|R(g)\|_{L^{r}\left(S^{3}\right)}$ is small enough, we can get good control of the Green's function $G$. Then since $\left(S^{3}, g\right)$ is convex near $P$, using the isoperimetric surfaces it can be shown that in at least a small region of the compact component of $\left(S^{3} \backslash\{P\}, G^{4} g\right)$, there is a surface on which the mean curvature points toward the asymptotically flat end. On the other hand, in the asymptotically flat end, we can always find a surface whose mean curvature points toward the compact component. Then by the standard existence theory in geometric measure theory there must be a minimal surface in between.

Proof of Theorem 1.2. Since we have the lower bound $\mu$ on the Ricci curvature and $S^{3}$ is compact hence complete, we have the following Poincaré inequality [5]

$$
\inf _{\beta \in \mathbf{R}} \int_{B_{\frac{R}{2}}}|f-\beta|^{2} \leq C_{2}(\mu) R^{2} \int_{B_{R}}|\nabla f|^{2}
$$

for any $f \in W_{1}^{2}\left(B_{R}\right)$.

By the Bishop comparison theorem we also have the upper volume bound

$$
\operatorname{Vol}\left(B_{R}, g\right) \leq V(\mu, R) \leq C_{3}(\mu, D) R^{3} \quad \text { for } R \leq D
$$

where $V(\mu, R)$ is the volume of the geodesic ball with radius $R$ in the manifold of constant curvature $\mu$. 
By a theorem of C. Croke [1], the isoperimetric constant of $S^{3}$

$$
\inf _{\Sigma} \frac{\operatorname{Area}(\Sigma, g)}{\min \left\{\operatorname{Vol}\left(M_{1}, g\right), \operatorname{Vol}\left(M_{2}, g\right)\right\}^{\frac{2}{3}}}
$$

(where $\Sigma$ runs over all the hypersurfaces which divide $S^{3}$ into $M_{1}$ and $M_{2}$ ) can be bounded below by some constant $C_{1}$ depending only on $V, D$ and $\mu$.

For $B_{R} \subset S^{3}$ with $\operatorname{Vol}\left(B_{R}\right)<\frac{1}{2} V \leq \frac{1}{2} V_{T}$

$$
\begin{aligned}
& \inf _{\Omega \subset B_{R}, \partial \Omega \cap \partial B_{R}=\emptyset} \frac{\operatorname{Area}(\partial \Omega, g)}{\operatorname{Vol}(\Omega, g)^{\frac{2}{3}}} \\
= & \inf _{\Omega \subset B_{R}, \partial \Omega \cap \partial B_{R}=\emptyset} \frac{\operatorname{Area}(\partial \Omega, g)}{\min \left\{\operatorname{Vol}(\Omega, g), \operatorname{Vol}\left(S^{3} \backslash \Omega, g\right)\right\}^{\frac{2}{3}}} \\
\geq & \inf _{\Sigma} \frac{\operatorname{Area}(\Sigma, g)}{\min \left\{\operatorname{Vol}\left(M_{1}, g\right), \operatorname{Vol}\left(M_{2}, g\right)\right\}^{\frac{2}{3}}} \\
\geq & C_{1} .
\end{aligned}
$$

Because [4]

$$
\inf _{f \in C^{1}\left(B_{R}\right), f=0 \text { on } \partial B_{R}} \frac{\int_{B_{R}}|\nabla f| d v_{g}}{\left(\int_{B_{R}}|f|^{\frac{3}{2}} d v_{g}\right)^{\frac{2}{3}}} \geq \inf _{\Omega \subset B_{R}, \partial \Omega \cap \partial B_{R}=\emptyset} \frac{\operatorname{Area}(\partial \Omega, g)}{\operatorname{Vol}(\Omega, g)^{\frac{2}{3}}},
$$

on $B_{R}$ the Sobolev inequality holds:

$$
C_{1}\left(\int_{B_{R}}|f|^{\frac{3}{2}} d v_{g}\right)^{\frac{2}{3}} \leq \int_{B_{R}}|\nabla f| d v_{g}
$$

for $f \in C^{1}\left(B_{R}\right)$ with $f=0$ on $\partial B_{R}$, and $C_{1}=C_{1}(V, D, \mu)$.

Therefore we can use Lemmas 2.1 and 2.2. in the proof.

We choose $\rho$ small to satisfy

$$
\operatorname{Vol}\left(B_{\rho}(P), g\right)<\frac{V}{2} \leq \frac{V_{T}}{2}
$$

and

$$
\text { Area }\left(\partial B_{\rho}(P), g\right)<(1+\epsilon)^{-10} I\left(S^{3}\right)\left(\frac{V}{2}\right) \leq(1+\epsilon)^{-10} I\left(S^{3}\right)\left(\frac{V_{T}}{2}\right)
$$

where $\epsilon>0$ is some fixed constant, and

$$
\begin{aligned}
& I\left(S^{3}\right) \\
= & \inf \left\{\frac{\operatorname{Area}(\Sigma)}{\min \left\{\operatorname{Vol}\left(M_{1}\right), \operatorname{Vol}\left(M_{2}\right)\right\}}: \quad \Sigma \text { divides } S^{3} \text { into } M_{1} \text { and } M_{2}\right\} .
\end{aligned}
$$

S.T.Yau [10] showed that $I\left(S^{3}\right)$ can be bounded below by $D, V$, and $\mu$. Note that $\rho$ only depends on $D, V, \mu$ and $\epsilon$. 
After scaling we can assume $G>1$ on $S^{3} \backslash B_{\frac{\rho}{2}}(P)$. We define the isoperimetric function as

$$
\begin{gathered}
A(V) \\
=\inf \left\{\operatorname{Area}\left(\partial \Omega, G^{4} g\right): \Omega \subseteq S^{3} \backslash B_{\frac{\rho}{2}}(P) \text { and } \operatorname{Vol}\left(\left(\Omega, G^{4} g\right)\right)=V\right\} .
\end{gathered}
$$

Lemma 3.1 below shows that there exists $0<\delta=\delta(V, D, \mu, r, \epsilon) \leq 1$ such that if $\|R(g)\|_{L^{r}\left(S^{3}\right)}<\delta$, then we can find appropriate $1<G<1+\epsilon$ on $S^{3} \backslash B_{\frac{\rho}{2}}(P)$.

Because $\operatorname{Vol}\left(S^{3} \backslash B_{\frac{\rho}{2}}(P), g\right)>\frac{V_{T}}{2}$, we know $\operatorname{Vol}\left(S^{3} \backslash B_{\frac{\rho}{2}}(P), G^{4} g\right)>\frac{V_{T}}{2}$. By an existence theorem in geometric measure theory [6], there exists a surface $\Sigma_{1} \subseteq S^{3} \backslash B_{\frac{\rho}{2}}(P)$ which realizes $A\left(\frac{V_{T}}{2}\right)$. That means $A\left(\frac{V_{T}}{2}\right)=\operatorname{Area}\left(\Sigma_{1}, G^{4} g\right)$ and $\operatorname{Vol}\left(\Sigma_{1}, G^{4} g\right)=\frac{V_{T}}{2}$, where $\operatorname{Vol}\left(\Sigma_{1}, G^{4} g\right)$ denotes the volume of the region in $S^{3} \backslash B_{\frac{\rho}{2}}(P)$ with $\Sigma_{1}$ as its boundary under metric $G^{4} g$.

Then since $G>1$,

$$
\begin{aligned}
A\left(\frac{V_{T}}{2}\right) & >\operatorname{Area}\left(\Sigma_{1}, g\right) \\
& >I\left(S^{3}\right) \cdot \min \left\{\operatorname{Vol}\left(\Sigma_{1}, g\right), V_{T}-\operatorname{Vol}\left(\Sigma_{1}, g\right)\right\}
\end{aligned}
$$

Because $1<G<1+\epsilon$ on $\Sigma_{1}$,

$$
\frac{1}{(1+\epsilon)^{6}} \frac{V_{T}}{2}=\frac{\operatorname{Vol}\left(\Sigma_{1}, G^{4} g\right)}{(1+\epsilon)^{6}}<\operatorname{Vol}\left(\Sigma_{1}, g\right)
$$

and

Then by (12)

$$
\operatorname{Vol}\left(\Sigma_{1}, g\right)<\operatorname{Vol}\left(\Sigma_{1}, G^{4} g\right)=\frac{V_{T}}{2}
$$

$$
A\left(\frac{V_{T}}{2}\right)>I\left(S^{3}\right) \cdot \operatorname{Vol}\left(\Sigma_{1}, g\right)>I\left(S^{3}\right) \cdot \frac{1}{(1+\epsilon)^{6}} \frac{V_{T}}{2}
$$

By the fact $1<G<1+\epsilon$ on $S^{3} \backslash B_{\rho}(P)$, we also have

$$
\begin{aligned}
\text { Area }\left(\partial B_{\rho}(P), G^{4} g\right) & <(1+\epsilon)^{4} \text { Area }\left(\partial B_{\rho}(P), g\right) \\
& <(1+\epsilon)^{-6} I\left(S^{3}\right) \cdot\left(\frac{V_{T}}{2}\right) \\
& <A\left(\frac{V_{T}}{2}\right)
\end{aligned}
$$

Therefore by the definition of the isoperimetric function

$$
\begin{aligned}
A\left(\operatorname{Vol}\left(S^{3} \backslash B_{\rho}(P), G^{4} g\right)\right) & \leq \text { Area }\left(\partial B_{\rho}(P), G^{4} g\right) \\
& <A\left(\frac{V_{T}}{2}\right) .
\end{aligned}
$$


Since

$$
\begin{aligned}
\operatorname{Vol}\left(S^{3} \backslash B_{\rho}(P), G^{4} g\right) & >\operatorname{Vol}\left(S^{3} \backslash B_{\rho}(P), g\right) \\
& >\frac{V_{T}}{2},
\end{aligned}
$$

and $A(V)$ is continuous, (13) implies that the function $A(V)$ attains its minimum on the interval $\left[\frac{V_{T}}{2}, \operatorname{Vol}\left(S^{3} \backslash B_{\rho}(P), G^{4} g\right)\right]$ at $V=V_{b}$ where $\frac{V_{T}}{2}<V_{b} \leq \operatorname{Vol}\left(S^{3} \backslash\right.$ $\left.B_{\rho}(P), G^{4} g\right)$.

Let $\Sigma_{b}=\partial \Omega_{b}$ be the surface which realizes $A\left(V_{b}\right)$. Since $\left(S^{3} \backslash\{P\}, G^{4} g\right)$ is almost Euclidean near $P$, we can find a surface $\Sigma_{\infty}$ outside of $\Sigma_{b}$ whose mean curvature points away from $P$, which means that the area will increase if we deform the surface in the direction pointing at $P$.

Let $U \subset S^{3}$ be the open subset bounded by $\Sigma_{\infty}$ and $\Sigma_{b}$. By the existence theory in geometric measure theory there is a surface $\Sigma \subset \bar{U}$ such that

$$
\begin{aligned}
\operatorname{Area}\left(\Sigma, G^{4} g\right)= & \inf \left\{\operatorname{Area}\left(\Sigma^{\prime}, G^{4} g\right):\right. \\
& \left.\Sigma^{\prime} \subset \bar{U}, \partial \Sigma^{\prime}=\emptyset \text { and } \Sigma^{\prime} \text { divides } \bar{U} \text { into two parts }\right\}
\end{aligned}
$$

We will show that $\Sigma$ is a smooth minimal surface. First of all, because $\Sigma$ is area-minimizing inside $\bar{U}$ and $\Sigma_{\infty}$ is strictly convex, we know that $\Sigma \cap \Sigma_{\infty}=\emptyset$. If also $\Sigma \cap \Sigma_{b}=\emptyset$, then $\Sigma$ is minimal since it is area-minimizing in $\bar{U}$ and doesn't touch the boundaries of $U$.

If $\Sigma$ does contact $\Sigma_{b}$, then let $\tilde{P} \in \Sigma \cap \Sigma_{b}$ and $B_{l}(\tilde{P})$ be a small ball centered at $\tilde{P}$ of radius $l$ under metric $G^{4} g$. Let $\Gamma=\left(\partial B_{l}(\tilde{P})\right) \cap \Sigma$, and let $\Sigma_{\Gamma}$ be the surface bounded by $\Gamma$ which minimizes area, i.e.,

$$
\operatorname{Area}\left(\Sigma_{\Gamma}, G^{4} g\right)=\min \left\{\operatorname{Area}\left(\hat{\Sigma}, G^{4} g\right): \partial \hat{\Sigma}=\Gamma\right\}
$$

We claim that $\Sigma_{\Gamma} \subseteq \bar{U}$. Suppose this is not true, then $\Sigma_{\Gamma} \cap \Omega_{b} \neq \emptyset$. Let $\tilde{\Sigma}=\left(\Sigma \backslash B_{l}(\tilde{P})\right) \cup \Sigma_{\Gamma}$, then $\tilde{\Sigma}=\partial \tilde{\Omega}$ for some region $\tilde{\Omega}$. By making the radius $l$ sufficiently small we have $\operatorname{Vol}\left(\Omega_{b} \cap \tilde{\Omega}, G^{4} g\right)>\frac{V_{T}}{2}$. Then since $A\left(V_{b}\right)$ is the minimum of the function $A(V)$ on $\left[\frac{V_{T}}{2}, V_{b}\right]$,

$$
A\left(\operatorname{Vol}\left(\Omega_{b} \cap \tilde{\Omega}, G^{4} g\right)\right)>A\left(V_{b}\right)=\operatorname{Area}\left(\partial \Omega_{b}, G^{4} g\right) .
$$

By the definition of $A(V)$ this implies that

$$
\text { Area }\left(\partial\left(\Omega_{b} \cap \tilde{\Omega}\right), G^{4} g\right)>\operatorname{Area}\left(\partial \Omega_{b}, G^{4} g\right) .
$$

This contradicts the minimizing property of $\Sigma_{\Gamma}$, thus $\Sigma_{\Gamma} \subseteq \bar{U}$. Then since $\Sigma$ is area-minimizing inside $\bar{U}$ and $\partial \Sigma_{\Gamma}=\Gamma \subset \Sigma$, we have $\Sigma_{\Gamma} \subseteq \Sigma$. Then because we also know $\Sigma \cap \Sigma_{\infty}=\emptyset, \Sigma$ is a minimal surface. This completes the proof.

Lemma 3.1. Same assumptions as those in Theorem 1.2. Let $u=G-1$. For any $\epsilon>0$ and $r>\frac{3}{2}$, there exists $0<\delta=\delta(V, D, \mu, \epsilon, r) \leq 1$, such that for our choice of $G$ we have $0<u<\epsilon$ on $S^{3} \backslash B_{\frac{\rho}{2}}(P)$ provided $\|R(g)\|_{L^{r}\left(S^{3}\right)}<\delta$. 
Proof. Let $R=\frac{\rho}{8}$, and let $\theta=\frac{1}{2}$. We can cover $S^{3} \backslash B_{\frac{\rho}{2}}(P)$ by balls centered on $S^{3} \backslash B_{\frac{\rho}{2}}(P)$ of radius $R$. Then each ball $B_{R}$ is away from the north pole $P$, and the enlarged balls $B_{\frac{R}{\theta}}$ is also away from $P$. On $B_{R}$, $u$ satisfies

$$
8 \triangle u-R(g) u=R(g)
$$

and $u>0$ after scaling.

On the one hand, $u$ satisfies $8 \triangle u-R(g) u>0$, so the standard elliptic theory implies that

$$
\sup _{B_{R}} u<C_{5}\left(R^{-3} \int_{B_{\frac{R}{\theta}}} u^{p} d v_{g}\right)^{\frac{1}{p}} \quad \text { for all } p>0
$$

where $C_{5}$ depends on $C_{1}, p, r, R$ and the upper bound on $\int_{B_{\frac{R}{\theta}}}|R(g)|^{r} d v_{g}$.

On the other hand, Lemma 2.1 shows that

$$
C_{6}\left(R^{-3} \int_{B_{\frac{R}{\theta}}} u^{p} d v_{g}\right)^{\frac{1}{p}} \leq \inf _{B_{R}} u+\left(\frac{R}{\theta}\right)^{2-\frac{3}{r}}\|R(g)\|_{L_{r}\left(B_{\frac{R}{\theta}}\right)}
$$

where $p$ and $C_{6}$ depend on $C_{1}, C_{2}, C_{3}, r, R$ and the upper bound on $\int_{B_{\frac{R}{\theta}}}|R(g)|^{r} d v_{g}$. Combining (15) and (16) we have

$$
\begin{aligned}
\sup _{B_{R}} u & <C_{7}\left(\inf _{B_{R}} u+R^{2-\frac{3}{r}}\|R(g)\|_{L_{r}\left(B_{\frac{R}{\theta}}\right)}\right) \\
& <C_{7}\left(\inf _{B_{R}} u+R^{2-\frac{3}{r}}\|R(g)\|_{L_{r}\left(S^{3}\right)}\right)
\end{aligned}
$$

where $C_{7}$ depends on $C_{1}, C_{2}, C_{3}, r, R$ as well as the upper bound on $\int_{B_{\frac{R}{\theta}}}|R(g)|^{r} d v_{g}$. Since we assume $\int_{S^{3}}|R(g)|^{r} d v_{g}<1, C_{7}$ depends on $C_{1}, C_{2}, C_{3}, r$ and $R$, hence only depends on $V, D, \mu, \epsilon$ and $r$.

We can cover $\Omega=S^{3} \backslash B_{\frac{\rho}{2}}(P)$ by finite small balls of radius $R$, such that the number of balls needed has an upper bound depending on $D, V$ and $R$, therefore on $D, V, \mu, \epsilon$ and $r$. To prove this, let $N$ be the maximal number of disjoint balls with radius $\frac{R}{2}$ on $S^{3}$, by the maximality balls with the same centers but radius $R$ will be able to cover $S^{3}$. Since these balls are disjoint,

$$
\sum_{i=1}^{N} \operatorname{Vol}\left(B_{\frac{R}{2}}\left(O_{i}\right), g\right)<V_{T}<C_{3}(\mu, D) D^{3}
$$

where $O_{i}$ are the centers of the balls.

We also know by the relative volume comparison theorem that

$$
\operatorname{Vol}\left(B_{\frac{R}{2}}\left(O_{i}\right), g\right) \geq C_{8}(D, V, R)=C_{8}(D, V, \mu, \epsilon, r) .
$$

Therefore $N \leq \frac{C_{3} D^{3}}{C_{8}}$ which only depends on $D, V, \mu, \epsilon, r$. 
Then by (17)

$$
\sup _{\Omega} u<C_{9}\left(\inf _{\Omega} u+\|R(g)\|_{L^{r}\left(S^{3}\right)}\right)
$$

where $C_{9}=C_{9}\left(\frac{C_{3} D^{3}}{C_{8}}, C_{7}, R, r\right)=C_{9}(V, D, \mu, \epsilon, r)$.

Let $\delta=\min \left\{1, \frac{\epsilon}{C_{9}}\right\}$, then $\delta$ only depends on $V, D, \mu, r$ and $\epsilon$. If $\|R(g)\|_{L^{r}\left(S^{3}\right)}<$ $\delta$, then we can always by scaling choose an appropriate $G$ such that $u>0$ and

$$
C_{9}\left(\inf _{\Omega} u+\|R(g)\|_{L^{r}\left(S^{3}\right)}\right)<\epsilon .
$$

Thus by (18) $\sup _{\Omega} u<\epsilon$.

\section{Acknowledgement}

This paper is based on part of the author's dissertation [9] at Stanford University. I would like to thank my advisor Prof. Richard Schoen for his help and support.

\section{References}

[1] C. B. Croke, Some isoperimetric inequalities and eigenvalue estimates, Ann. Sci. École Norm. Sup. (4), 13 (1980), 419-435.

[2] D. M. Eardley, Gravitational collapse of vacuum gravitational field configurations, J. Math. Phys., 36 (1995), 3004-3011.

[3] J. L. Kazdan and F. W. Warner, Scalar curvature and conformal deformation of Riemannian structure, J. Differential Geometry, 10 (1975), 113-134.

[4] P. Li, Lecture notes on geometric analysis, volume 6 of Lecture Notes Series, Seoul National University Research Institute of Mathematics Global Analysis Research Center, Seoul, 1993.

[5] R. Schoen, Lecture notes on PDEs, Stanford University.

[6] A lower bound for the first eigenvalue of a negatively curved manifold, J. Differential Geom., 17 (1982), 233-238.

[7] R. Schoen and S. T. Yau, The existence of a black hole due to condensation of matter, Comm. Math. Phys., 90 (1983), 575-579.

[8] R. M. Schoen, Variational theory for the total scalar curvature functional for Riemannian metrics and related topics, In Topics in calculus of variations (Montecatini Terme, 1987), volume 1365 of Lecture Notes in Math., 120-154. Springer, Berlin, 1989.

[9] Y. Yan, Some geometric problems involving conformal deformation of metrics, Dissertation, Stanford University, 2004.

[10] S. T. Yau, Isoperimetric constants and the first eigenvalue of a compact Riemannian manifold, Ann. Sci. École Norm. Sup. (4), 8 (1975), 487-507.

Department of Mathematics, The University of British Columbia, Vancouver, B.C., V6T 1Z2, CANAdA

E-mail address: yyan@math.ubc.ca 